\title{
XLII. On the composition and resolution of forces
}

\section{G.W. Hearn Esq. B.A.}

To cite this article: G.W. Hearn Esq. B.A. (1846) XLII. On the composition and resolution of forces, Philosophical Magazine Series 3, 29:194, 258-261, DOI: $10.1080 / 14786444608645502$

To link to this article: http://dx.doi.org/10.1080/14786444608645502

曲 Published online: 30 Apr 2009.

Submit your article to this journal $2 \pi$

Џ Article views: 2

Q View related articles $₫$ 
the corresponding rising of the effect of that force in the glass. M. E. Becquerel does not agree with me in the alove expla. nation, but thinks it is due to this; that the particles of the diamagnetic itself require time to assume their new state. That they may require time is, I think, very probable. I do not know any state the acquiring of which does not need time. This time is however most probably exceedingly small in the instance of diamagnetic bodies; and that the phænomena referred to by me are not due to such a cause, is, I think, shown by two considerations. The first is, that the electromagnet is well known by other kinds of proof, as the induction of currents, \&c., to require time to develope its maximum force due to a given electric current. I have shown that the rotation of the ray must increase during the time the magnet is thus rising; and $I$ found that the power to induce currents exists simultaneously with the increasing rotation. The next consideration is, that when the diamagnetic is submitted, not to the action of an electro-magnet, but of a helix, the rotation does not increase in the same gradual manner as before, but is instantly at a maximum (Experimental Researches, 2195): hence my reasons for adhering to the explanation I gave in the paper's themselves last year.

But on subjects so new as these, differences of opinion must inevitably arise upon many points; and it is better for the inquiries themselves that it should be so, for the facts in consequence receive a more close investigation. I therefore leave many points of difference between myself and others unnoticed for the present; believing that new and connected facts will rapidly accumulate, and that time, with his powerful aid, will in a very few years give both facts and opinions their right places.

Royal Institution, Angust 11, 1846.

XLII. On the Composition and Resolution of Forces. By G. W. Henrn, Esq., B.A. of Cambridge, and Professor of Mathematics in the Royal Military College, Sandhurst*

1. EQUAL forces are such as being applied to the same 1 point in contrary directions make equilibrium.

2. The resultant of any number of forces acting on a point is a single force, statically equivalent to the whole of them, that is a force such as would produce the same pressure in the same direction.

3. If two equal forces inclined to each other act on a point, their resultant is in the plane of the two forces, and bisects

* Communicated by the Author. 
the angle formed by their directions; and half that resuliant is defined to be the 'resolvent' of either of the equal forces.

4. When any number of forces act on a point, if any straight line be drawn through that point, and planes be drawn through this line and the respective directions of the forces; and if in each plane a force equal to the force in that plane be applied to the point, making an angle with the straight line equal to that which the other force makes, but on the contrary side of the line, such a system of forces is called 'supplementary' to the former.

5. A system of forces acting on a point is in equilibrium when their resultant is zero.

Prop. I. When a system of forces is in equilibrium, the sum of the resolvents in the direction of any straight line through their point of application is zero.

The system being in equilibrium, the supplementary system will also be in equilibrium; for this latter system is nothing more than the former turned through $180^{\circ}$ round the assumed line.

The two systems are therefore also in equilibrium, and consequently the resultant zero. But this resultant consists of the (algebraic) sum of the several resultants of the pairs of equal forces in the several planes through the assumed line, and half the resultant is therefore the sum of the resolvents of the given system. The resultant being zero, the sum of the resolvents is also zero.

Prop. II. Denoting the resolvent of a force $\mathbf{P}$ in the direction of a line making an angle $\theta$ with the direction of the force by $\mathrm{P} f \theta$, it is required to assign the form of $f \theta$.

It is an immediate consequence of our definition of resolvent that $f \theta=1$ when $\theta=0$, and that $f \theta=0$ when $\theta=\frac{\pi}{2}$. Moreover, that $f \theta$ cannot $=0$ for any value of $\theta<\frac{\pi}{2}$.

Also that $f^{\prime} \theta$ is a periodical function going through all its values, as $\theta$ increases from 0 to $2 \pi$; and that $f \theta$ can never be infinite, and must have some determinate single value for every value of $\theta$, and is therefore a function which can be expanded in integral positive powers of $\theta$.

Let two equal forces keep a point in equilibrium. Sum of resolvents $=0$ gives

or

$$
\begin{gathered}
\mathrm{P} f^{\theta}+\mathrm{P} f(\pi+\theta)=0, \\
f \theta+f(\pi+\theta)=0 .
\end{gathered}
$$

This equation is easily resolved, but is too general for our present purpose. 
To obtain further information as to the form of $f \theta$, let $\mathrm{P}, \mathrm{Q}$, $\mathbf{R}$ be three forces in equilibrium, and therefore necessarily in the same plane, and let a line be drawn through their point of application also in the same plane, inclined at angles $\theta$, $\alpha+\theta, \beta+\theta$ to $\mathbf{P}, \mathbf{Q}, \mathbf{R}$ respectively, then sum of resolvents $=0$ gives

$$
P f^{\prime} \theta+Q f(\alpha+\theta)+R f(\beta+\theta)=0
$$

for all values of $\theta$.

On equating to zero the coefficients of the several powers of $\theta$, we have the conditions

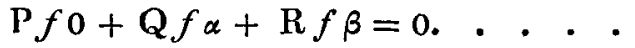

$$
\begin{aligned}
& \mathrm{P} f^{\prime} 0+\mathrm{Q} f^{\prime} \alpha+\mathrm{R} f^{\prime} \beta=0 . . . \text {. } \\
& \mathrm{P} f^{\prime \prime} 0+\mathrm{Q} f^{\prime \prime} \alpha+\mathrm{R} f^{\prime \prime} \beta=0 \text {. . . . . } \\
& \mathbf{P} f^{\prime \prime \prime} 0+\mathbf{Q} f^{\prime \prime \prime} \alpha+\mathbf{R} f^{\prime \prime \prime} \beta=0 . . . \\
& \text { \&c. } \\
& \text { \&c. }
\end{aligned}
$$

But the ratios $\frac{\mathrm{Q}}{\mathrm{P}}, \frac{\mathrm{R}}{\mathrm{P}}$ must be determinate functions of $\alpha$ and $\beta$, since the forces proportionally altered will still be in equilibrium. Hence the preceding conditions must be equivalent to two only.

Now if (1.) and (2.) were identical, or equivalent equations, all the rest would be equivalent to them; since 3 is derived from 2 in the same manner as 2 from $1, \& c$. The whole set would then reduce to only one, which is insufficient for the determination of $\frac{\mathrm{Q}}{\mathrm{P}}, \frac{\mathrm{R}}{\mathrm{P}}$.

Hence (3.) must be a consequence of (1.) and (2.), so that if $\lambda$ and $\mu$ be certain determinate constants, we have

$$
\begin{aligned}
& f^{\prime \prime} \alpha+\lambda f^{\prime} \alpha+\mu f \alpha=0, \\
& f^{\prime \prime} \beta+\lambda f^{\prime} \beta+\mu f \beta=0 .
\end{aligned}
$$

And since these conclusions are independent of any specific values of $\alpha$ and $\beta$, we have generally

$$
f^{\prime \prime} \theta+\lambda f^{\prime} \theta+\mu f \theta=0 \text {. }
$$

And it is easy to see that from this (4.) will be a consequence of (2.) and (3.), \&c., and the whole system of conditions will be equivalent to two only, videlicet the two first.

Now if $a$ and $b$ be the roots of

the solution of $(A$.$) is$

$$
x^{2}+\lambda x+\mu=0
$$

$$
f \theta=\mathrm{A} e^{a \theta}+\mathrm{B} e^{b \theta}
$$


On Substances contained in the Roccella tinctoria. 261

but since $f \theta$ must be periodical, $a$ and $b$ will be imaginary; let therefore $a=m+n \sqrt{-1}$ and $b=m-n \sqrt{-1}$. Then

$$
f \theta=e^{m \theta}\{\mathrm{A} \cos n \theta+\mathrm{B} \sin n \theta\} ;
$$

and that this may be periodical $m$ must $=0$. Also, since

$$
f \theta+f(\pi+\theta)=0,
$$

$n$ is restricted to be an odd integer.

Also when $\theta=0 f \theta=1, \therefore A=1$; and when $\theta=\frac{\pi}{2} f \theta=0$.

But $n$ being odd $\cos n \frac{\pi}{2}=0 \sin n \frac{\pi}{2}= \pm 1 . \quad$ Hence $B=0$, and therefore

$$
f^{\theta}=\cos n \theta
$$

but $\cos n \theta=0$ when $n \theta=\frac{\pi}{2}$, or $\theta=\frac{\pi}{2 n} ;$ but $f \theta$ cannot $=0$, for $\theta<\frac{\pi}{2}$. Hence $n$ is restricted to be $=1$, and therefore, finally,

$$
f \theta=\cos \theta .
$$

XLIII. On the Substances contained in the Roccella tinctoria. By Edward Schunck, Esq.*

THE Roccella tinctoria derives its interest from the fact of 1 its being that species of lichen from which the finest kind of archil is prepared. It has been examined by Heeren and Kane. The former discovered in it a peculiar substance, which he called Erythrine, and a fat acid named by him Roccellic acid. The latter extracted from the plant a substance which he called Erythriline, similar in properties to Heeren's erythrine, and a body to which he applied the name of Erythrine, but which possesses all the properties of the substance called by Heeren Pseuderythrine, and supposed by him to be a product of the action of boiling alcohol on his erythrine My results do not agree entirely with those of either of these chemists.

The lichen which I employed for my investigation was obtained from Angola and Madagascar, and was pronounced by a distinguished botanist, to whom I submitted it, to be the Roccella tinctoria var. fuciformis. In order to extract the various substances contained in it, I submitted it to the following operations :1846.

Communicated by the Chemical Society; having been read April 6 , 